\title{
Endocan expression correlated with total volume of coronary artery dilation in patients with coronary artery ectasia
}

\author{
Sylwia Iwańczyk, Aleksander Araszkiewicz, Michał Borger, Mikołaj Kamiński, Michał Wrotyński, \\ Ewa Chmara, Artur Cieślewicz, Artur Radziemski, Maciej Lesiak
}

$1^{\text {st }}$ Department of Cardiology, Poznan University of Medical Sciences, Poznan, Poland

Adv Interv Cardiol 2020; 16, 3 (61): 294-299

DOI: https://doi.org/10.5114/aic.2020.99264

\begin{abstract}
A bstract
Introduction: Inflammation and angiogenesis disturbances are considered as factors contributing to the development of coronary artery ectasias (CAE). Endocan (endothelial cell-specific molecule-1 - ESM-1) regulates both inflammatory and angiogenetic processes. However, there are no data about the correlation between endocan level and the severity of CAE measured with total volume of coronary artery dilation.

Aim: To assess whether the severity of the inflammatory process measured as endocan concentration correlates with the total volume of CAE.

Material and methods: We selected prospectively a total of 43 consecutive patients with coronary artery ectasia from 2240 patients who underwent coronary angiography in our center. Determination of endocan was performed by using the Human Endothelial cell-specific Molecule 1 (ECSM1/ENDOCAN) ELISA Kit. 3D QCA (three-dimensional quantitative coronary angiography) was used for coronary lesion and aneurysm quantification. The total volume of dilation was defined as the volume of all aneurysms and ectasias of coronary arteries in 1 patient.

Results: The mean volume of all aneurysms in 1 patient was $677 \pm 878.7 \mathrm{~mm}^{3}$. The total aneurysm volume was positively strongly correlated with endocan concentration (Pearson correlation coefficient: 0.811 ; 2-tailed $p<0.001$ ).

Conclusions: Endocan is a potential marker of vascular wall damage mainly as a result of inflammation in the course of atherosclerosis, but also vascular remodeling as a result of a disturbance of pro- and anti-angiogenic processes. Endocan level reflects the intensity of the above processes and therefore correlates with the severity of CAE, measured as the total volume of dilation.
\end{abstract}

Key words: inflammation, coronary atherosclerosis, coronary aneurysms, angiogenic proteins.

S u m m a ry

Endocan (endothelial cell-specific molecule-1 - ESM-1) regulates major physiologic and pathophysiologic processes, such as cell adhesion, inflammation, tumor progression, and angiogenesis, and probably reflects the degree of endothelial dysfunction. However, there are no data about the correlation between endocan level and the severity of coronary artery dilation. We found a positive correlation between endocan plasma level and the total volume of aneurysms, and thus the severity of atherosclerosis and angiogenesis. Reduction of the severity of both processes may affect the further development of coronary artery ectasias (CAE). However, further research is needed to understand the pathogenesis of CAE better.

\section{Introduction}

Coronary artery ectasia (CAE) is a dilation of the coronary artery lumen, exceeding the largest diameter of an adjacent normal vessel more than 1.5 fold [1]. CAE is a relatively uncommon finding encountered during diag- nostic coronary angiography. The incidence varies between $0.3 \%$ and $4.9 \%$ [2]. However, the rate of detection has increased during the last years, probably due to the introduction of new diagnostic modalities, such as magnetic resonance or computed tomography [3-5]. The pathophysiology of CAE remains unclear, although

\section{Corresponding author:}

Sylwia Iwańczyk, Department of Cardiology, Poznan University of Medical Sciences, 1/2 Długa St, 61-848 Poznan, Poland,

e-mail: syl.iwanczyk@gmail.com

Received: 16.02.2020, accepted: 7.05.2020. 
several mechanisms are currently suggested (Table I) [2]. Arterial remodeling in the course of atherosclerosis may be bidirectional according to the expansion or shrinkage of media and external elastic membrane [6]. This phenomenon is cold positive and negative remodeling, respectively. Positive remodeling is principally a compensatory mechanism to preserve luminal size during the progression of atherosclerosis [7]. In turn, the extensive remodeling in which both external elastic membrane and luminal size increase can contribute to CAE development $[8,9]$. However, only in a small proportion of patients does it lead to the formation of aneurysms. Inflammation is considered as one of the factors contributing to the development of CAE, which is closely related to the process of atherogenesis [10-12]. Probably, the intensity of this process and the type of inflammatory response determine different clinical entities, including plaque rupture, coronary spasm, coronary microvascular dysfunction, silent myocardial ischemia, restenosis, and finally coronary artery dilatation. Angiogenesis may also play a role in the pathogenesis of CAE. The occurrence of increased angiogenetic activity suggests accelerated and persistent extracellular matrix remodeling which predisposes to aneurysm formation [13].

Endocan (endothelial cell-specific molecule-1-ESM-1) is a proteoglycan produced predominantly by activated endothelial cells. It regulates major physiologic and pathophysiologic processes, such as cell adhesion, inflammation, tumor progression, and angiogenesis, and probably reflects the degree of endothelial dysfunction

Table I. Causes of coronary artery ectasia

\begin{tabular}{|c|c|}
\hline Etiology & $\begin{array}{l}\text { Approximate } \\
\text { frequency }\end{array}$ \\
\hline Atherosclerosis & $50 \%$ \\
\hline Kawasaki disease & $17 \%$ \\
\hline $\begin{array}{l}\text { Infections: infective endocarditis, (septic blockag- } \\
\text { es - mycotic aneurysms), syphilis, Lyme disease }\end{array}$ & $11 \%$ \\
\hline CTD (systemic lupus erythematosus and others) & $10 \%$ \\
\hline Vasculitis: polyarteritis nodosa, Takayasu disease & $10 \%$ \\
\hline Marfan disease & $<1 \%$ \\
\hline Ehlers-Danlos syndrome & $<1 \%$ \\
\hline Rendu-Osler-Weber syndrome & $<1 \%$ \\
\hline Neurofibromatosis type 1 & $<1 \%$ \\
\hline Congenital & $<1 \%$ \\
\hline Cancers & $<1 \%$ \\
\hline Thoracic injuries & $<1 \%$ \\
\hline $\begin{array}{l}\text { latrogenic, e.g., PCI, DES, atherectomy, rotabla- } \\
\text { tion, laser ablation, coronary brachytherapy }\end{array}$ & $<1 \%$ \\
\hline
\end{tabular}

due to a systemic inflammatory response [14]. However, there are no data about the correlation between endocan level and the severity of coronary artery dilation.

\section{Aim}

We sought to assess whether the intensity of the inflammatory process measured as endocan concentration correlates with the total volume of CAE.

\section{Material and methods}

We selected prospectively a total of 43 consecutive patients with coronary artery ectasia from 2240 patients who underwent coronary angiography in our catheterization laboratory between January 2017 and November 2017, due to typical angina pain and/or positive results of a non-invasive test suggesting the presence of myocardial infarction. Coronary artery ectasia was diagnosed when a coronary diameter exceeded $\geq 1.5$ times the original caliber of the artery or the adjacent segment diameter. We distinguish the following morphological types: sagittal when the transverse dimension is larger than the longitudinal dimension, and fusiform - when the longitudinal size is larger than transverse dimension [15]. The classification system proposed by Markis et al. additionally includes the number and diffuseness of involved coronary vessels (Table II) [16].

Exclusion criteria were as follows: acute inflammatory disorder (hsCRP > $10 \mathrm{mg} / \mathrm{l}$ ), active neoplastic disease, systemic diseases of connective tissue, treatment with interferon, thrombotic diathesis and plasmatic diathesis, acute renal failure or chronic renal failure with glomerular filtration rate (GFR) $<30 \mathrm{ml} / \mathrm{min}$, allergy to iodinated contrast medium, as well as lack of consent.

We evaluated data on clinical presentation, precipitating factors, cardiovascular risk factors, medications, and demographics as well. The study adhered to the requirements of the local ethics committee. All patients provided written informed consent. Coronary angiography and evaluation of coronary arteries were performed by experienced operators (the number of completed examinations >1000), not linked to the study. Because arterial aneurysmal disease is often a multilevel disease, all CAE patients were also screened for an aortic aneurysm.

Blood samples on the first day after coronary angiography were obtained. For blood serum collection stan-

Table II. Markis classification of vessel involvement

\begin{tabular}{|c|c|}
\hline Types of CAE & Definition \\
\hline 1 & Diffuse ectasia of two or three vessels \\
\hline 2 & $\begin{array}{l}\text { Diffuse ectasia in one vessel and localized disease } \\
\text { in another }\end{array}$ \\
\hline 3 & Diffuse ectasia in one vessel only \\
\hline 4 & Localized or segmental involvement \\
\hline
\end{tabular}


dard tubes with a chemically neutral coagulation activator (the main component is $\mathrm{SiO}_{2}$ ) were used. In the serum routine laboratory parameters were determined. The remainder of the sera was aliquoted, frozen and stored at $-80^{\circ} \mathrm{C}$.

Determination of endocan was performed using the Human Endothelial cell-specific Molecule 1 (ECSM1/ ENDOCAN) ELISA Kit. This ELISA kit is based on the principle of double-antibody sandwich technique to detect Human ECSM1/ENDOCAN manufactured by DRG MedTek company. The sensitivity of this assay was determined by sub-tracing two standard deviations to the mean optical density value of twenty zero standard replicates and calculating the corresponding concentration. The assay range was between 8 and $2000 \mathrm{ng} / \mathrm{l}$. The analysis was performed in the laboratory of clinical pharmacology.

3D QCA (three-dimensional quantitative coronary angiography) was used for coronary aneurysm quantification using dedicated software QANGIO XA 3D RE straight Medis, Leiden, Netherlands. The 3D QCA system combines information from 2D angiographic projections to calculate a $3 \mathrm{D}$ reconstruction and perform quantitative analysis on the vessel part of interest, such as length, diameters, cross-sectional areas and the volume of aneurysm (Figure 1). The differences in angulation between the analyzed projections should be at least $30^{\circ}$. The operator performs a semi-automatic 2D contour detection in both projections. A common image point (CIP) is auto- matically placed at a similar position in both projections and its position can be modified by the operator. The total volume of dilation was defined as the volume of all aneurysms and ectasias of coronary arteries in 1 patient.

\section{Statistical analysis}

Statistical analysis was performed with Statistica 12.0 (StatSoft, USA). Normality of the variables' distributions was tested using the Shapiro-Wilk test. The report includes descriptive analysis, the Kruskal-Wallis test, ANOVA test, and Mann-Whitney $U$ test to compare study groups. The association between continuous parameters were tested by Pearson correlation analysis. Linear regression models were deployed to study correlations between endocan level and total aneurysm volume. Differences with $p$-value $<0.05$ were considered statistically significant.

\section{Results}

The baseline clinical characteristics are presented in Table III. Mostly, we observed CAE in a middle-aged man with hypertension, overweight, and hyperlipidemia. About one third had diabetes, and almost $40 \%$ of patients have had a previous myocardial infarction. Seven (16.3\%) patients were diagnosed with abdominal aortic aneurysm (AAA).

CAEs were most often located in the right coronary artery (RCA), including the whole vessel as well as limited
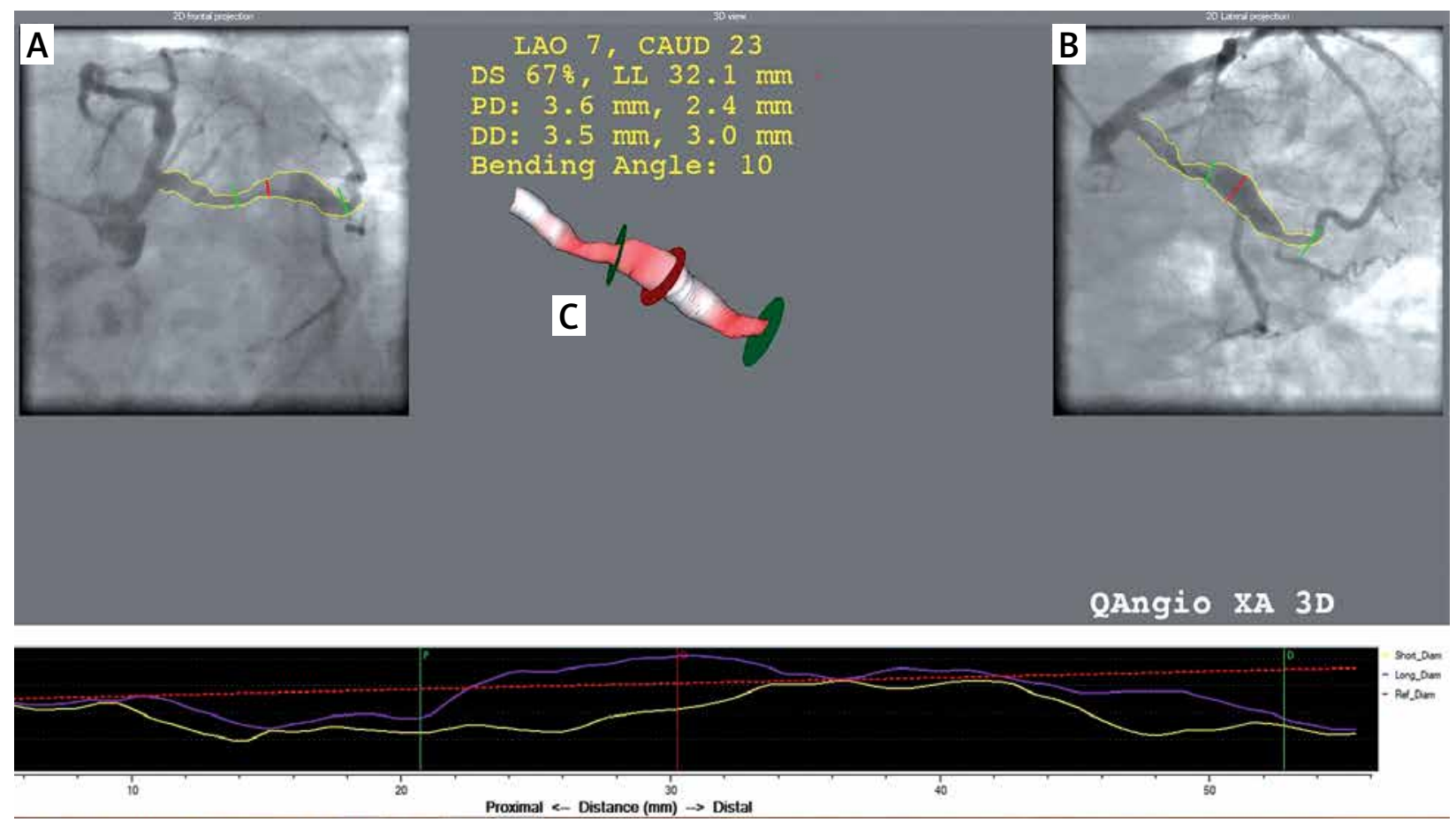

Figure 1. 3D reconstruction $(\mathbf{C})$ based on $2 \mathrm{D}$ angiographic projections ( $\mathbf{A}$ and $\mathbf{B})$. The red line in image $\mathbf{A}$ and $\mathbf{B}$ is the common image point and determines the point of maximum short and long diameter. The green lines mark the boundaries of the aneurysm 
Table III. Baseline clinical characteristics, means \pm SD or $n(\%)$

\begin{tabular}{lc} 
Characteristics & CAE \\
\hline$N$ & 43 \\
\hline Male & $34(79.1)$ \\
\hline Age [years] & $66.4 \pm 8.7$ \\
\hline BMI [kg/m²] & $29.7 \pm 7.8$ \\
\hline CAD (yes/no) & $26(60.5)$ \\
\hline Hypertension (yes/no) & $35(81.4)$ \\
\hline Diabetes (yes/no) & $15(34.9)$ \\
\hline Past history of MI (yes/no) & $17(39.5)$ \\
\hline Hyperlipidemia & $20(46.5)$ \\
\hline AAA & $7(16,3)$
\end{tabular}

$A A A$ - abdominal aortic aneurysm, $B M I$ - body mass index, $C A D$ - coronary artery disease, CAE - coronary artery ectasia, MI-myocardial infarction.

to the proximal or middle section, and in the left anterior descending artery (LAD), most often in the proximal segment (Table IV). Diffuse ectasias were significantly more frequent than localized aneurysms. The extent of coronary artery involvement according to the Markis classification is presented in Table $V$. Mixed form, including localized and diffuse disease, was the rarest. The other types occurred with similar frequency. Significant stenosis of coronary arteries was diagnosed in $60.5 \%$ of patients with CAE. In this group localized aneurysms dominated (44.4\%), while in the non-coronary artery disease (non-CAD) group diffuse ectasias occurred more often (81.3\%). The number of vessels involved and localization were similar for both groups (Table VI).

The mean volume of all aneurysms in 1 patient was $677 \pm 878.7 \mathrm{~mm}^{3}$. Linear regression analysis revealed a correlation between endocan level and total aneurysm volume (Figure 2 ). The total aneurysm volume was strongly positively correlated with endocan concentration (Pearson correlation coefficient: 0.811; 2-tailed $p<0.001)$.

\section{Discussion}

We found a positive correlation between endocan plasma level and the total volume of aneurysms. Endo-

Table V. Extent of vessel involvement according to Markis classification

\begin{tabular}{lc} 
Type & $\boldsymbol{N}(\%)$ \\
\hline 1 & $13(30.2)$ \\
\hline 2 & $3(7.0)$ \\
\hline 3 & $13(30.2)$ \\
\hline 4 & $14(32.6)$
\end{tabular}

Table IV. Vessel localization of CAE

\begin{tabular}{lc} 
Vessel & $\boldsymbol{N}(\%)$ \\
\hline$R C A:$ & $23(53.5)$ \\
\hline WRCA & $9(39.1)$ \\
\hline pRCA & $8(34.8)$ \\
\hline mRCA & $7(30.4)$ \\
\hline dRCA & 0 \\
\hline LCX: & $16(37.2)$ \\
\hline WLCX & $4(25.0)$ \\
\hline pLCX & $10(62.5)$ \\
\hline dLCX & $2(8.7)$ \\
\hline LAD: & $21(48.8)$ \\
\hline WLAD & $2(9.5)$ \\
\hline pLAD & $17(81.0)$ \\
\hline mLAD & $2(8.7)$ \\
\hline dLAD & 0
\end{tabular}

$L A D$ - left anterior descending, $L C X$ - left circumflex, $L M$ - left main, $R C A$ - right coronary artery, $d$-distal, $m$ - middle, $p$-proximal, $w$ - whole.

can is strongly associated with inflammation and endothelial dysfunction, which are the primary pathologies in the development of atherosclerosis - the most common cause of aneurysm in adults. Moreover, endocan is an important mediator of angiogenesis, disturbances of which are also considered to be a possible mechanism of CAE development [17-19].

Table VI. Comparison of angiographic data of patients with and without significant artery stenosis

\begin{tabular}{lcc} 
Characteristics & CAD & Non-CAD \\
\hline$N$ & 27 & 16 \\
\hline Diffuse ectasia & $13(48.1)$ & $13(81.3)^{*}$ \\
\hline Localized disease & $12(44.4)$ & $2(12.5)^{*}$ \\
\hline Both & $2(7.4)$ & $1(6.3)$ \\
\hline Number of vessels involved: & & \\
\hline 1 & $17(63.0)$ & $9(56.3)$ \\
\hline 2 & $6(22.2)$ & $5(31.3)$ \\
\hline 3 & $4(14.8)$ & $2(12.5)$ \\
\hline LM & $4(14.8)$ & $1(6.3)$ \\
\hline RCA & $14(51.9)$ & $9(56.3)$ \\
\hline LAD & $12(44.4)$ & $9(56.3)$ \\
\hline LCX & $10(37.0)$ & $6(37.5)$
\end{tabular}

$C A D$ - coronary artery disease, $L A D$ - left anterior descending, $L C X$ - left circumflex, $L M$ - left main, $R C A$ - right coronary artery, ${ }^{*} p<0.05$. 


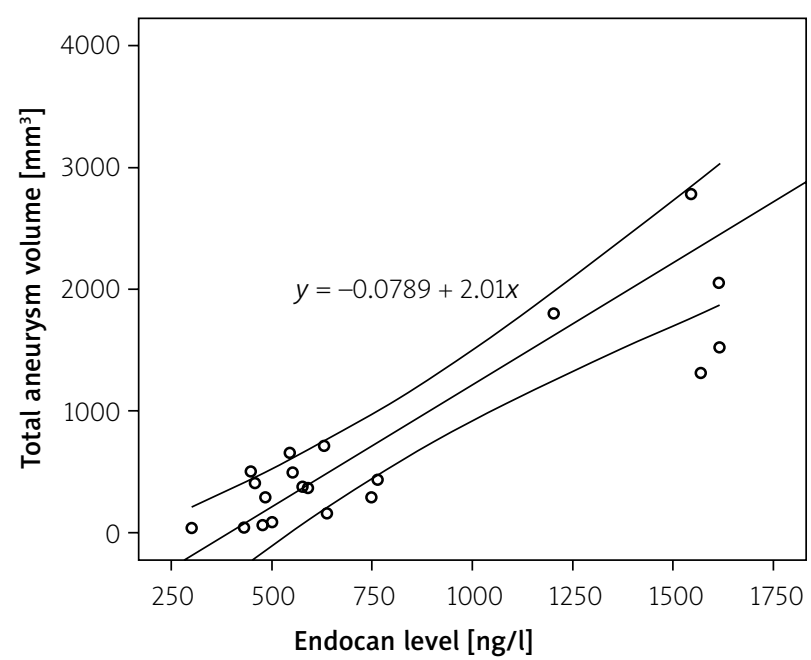

Figure 2. Linear regression correlation between endocan level and total aneurysm volume

Our results showed that more than half of the patients with CAE also had coronary artery disease. Histological changes in both CAE and atherosclerotic lesions are very similar and include diffuse hyalinization, intimal and medial damage [20]. The risk factors of atherosclerosis, such as increased lipid accumulation, elevated homocysteine, increased insulin and NO metabolites, genetic predisposition, enhanced activity of renin-angiotensin system, promote the expression and activity of matrix-degrading enzymes. It causes severe disruption of the internal elastic lamina and intensifies inflammation in the media, favoring excessive expansive remodeling, which increases the risk of coronary ectasia formation.

As a response to endothelial injury, the immune-inflammatory reaction occurs and, as a consequence, the chronic inflammatory process develops [21]. Li et al. hypothesized that CAE might be associated with inflammation. They observed significantly higher levels of hs-CRP in CAE compared to coronary artery disease (CAD) [22]. A significant correlation between high-sensitivity C-reactive protein (hs-CRP) level and length of ectasia was also found. Turan et al. observed that endocan serum level was significantly higher in the isolated CAE population in comparison to the control group. Moreover, they found a significant correlation between endocan levels and severity of isolated CAE measured by the Markis classification [23]. Additionally, many studies showed increased expression of different inflammatory factors, such as vascular cell adhesion molecule-1 (VCAM-1), intercellular adhesion molecule-1 (ICAM-1) and E-selectin, which facilitate the adhesion and migration of the neutrophils and mononuclear cells across the vascular endothelial layer [24, 25].

Another possible pathomechanism of CAE is the angiogenesis process coexisting with arteriosclerosis. Hypoxia, which arises as a result of significant atherosclerotic changes, activates vascular remodeling and formation of new blood vessels from the existing ones. Angiogenesis involves several stages and is regulated by many molecular mechanisms based on the balance between pro- and anti-angiogenic factors [26, 27]. One of the potential regulatory factors is endocan. The data regarding the influence of angiogenesis on the pathogenesis of coronary artery ectasia in adults are insufficient. Only single reports have shown increased secretion of such factors as vascular endothelial growth factor (VEGF) in patients with diffuse CAE [7]. However, the contribution of angiogenesis to the pathomechanism of aneurysms in Kawasaki disease is well documented, both in the acute period and in long-term observation $[8,9]$.

In addition, the analysis showed increased incidence of aortic aneurysms in the study group (16.3\%). All of them concerned the abdominal segment. In the general population, ultrasound screening shows abdominal aortic aneurysm in $4.4 \%$ of people aged $40-75$. However, no data are available regarding the relationship between endocan and AAA pathogenesis.

\section{Conclusions}

Endocan is a potential marker of vascular wall damage mainly as a result of inflammation in the course of atherosclerosis, but also vascular remodeling as a result of a disturbance of pro- and anti-angiogenic processes. Endocan level reflects the intensity of the above processes and therefore correlates with the severity of CAE, measured with the total volume of dilation. Reduction of the severity of both processes may affect the further development of CAE. Undoubtedly, further research is needed to understand the pathogenesis of CAE better.

\section{Conflict of interest}

The authors declare no conflict of interest.

\section{References}

1. Swaye PS, Fisher LD, Litwin P, et al. Aneurysmal coronary artery disease. Circulation 1983; 67: 134-8.

2. Araszkiewicz A, Grygier M, Lesiak $M$, et al. Review article From positive remodelling to coronary artery ectasia. Is coronary artery aneurysm a benign form of coronary disease? Kardiol Pol (Pol Heart J) 2009; 67: 1390-5.

3. Leschka S, Alkadhi H, Plass A, et al. Accuracy of MSCT coronary angiography with 64-slice technology: first experience. Eur Heart J 2005; 26: 1482-7.

4. Greil GF, Stuber M, Botnar RM, et al. Coronary magnetic resonance angiography in adolescents and young adults with Kawasaki disease. Circulation 2002; 105: 908-11.

5. Mavrogeni S, Papadopoulos G, Douskou M, et al. Magnetic resonance angiography is equivalent to $X$-ray coronary angiography for the evaluation of coronary arteries in Kawasaki disease. J Am Coll Cardiol 2004; 43: 649-52.

6. Mintz GS, Paanter JA, Pichard AL, et al. Atherosclerosis in angiographically "normal" coronary artery reference segments. J Am Coll Cardiol 1995; 25: 1479-85. 
7. Glagov S, Weisenberg E, Zarins CK, et al. Compensatory enlargement of human atherosclerotic coronary arteries. N Engl I Med 1987; 316: 1371-53.

8. Bergland H, Ham CJ, Siegel RT. Contribution of inadequate compensatory enlargement to development of human artery stenosis: an in vivo intravascular study. J Am Coll Cardiol 1996; 27 : 1571-76.

9. Pasterkamp G, Wensing PJW, Post MJ, et al. Paradoxical arterial wall shrinkage may contribute to luminal narrowing of human atherosclerotic femoral arteries. Circulation 1995; 91: 1444-9.

10. Galis ZS, Sukhova GK, Lark MW, et al. Increased expression of matrix metalloproteinases and matrix degrading activity in vulnerable regions of human atherosclerotic plaques. J Clin Invest 1994; 94: 2493-503.

11. Shah PK, Falk E, Badimon JJ, et al. Human monocyte-derived macrophages induce collagen breakdown in fibrous caps of atherosclerotic plaques. Circulation 1995; 92: 1565-9.

12. Henney AM, Wakeley PR, Davies MJ, et al. Localization of stromelysin gene expression in atherosclerotic plaques by in situ hybridization. Proc Natl Acad Sci USA 1991; 88: 8154-8.

13. Savino M, Parisi Q, Biondi-Zoccai GG, et al. New insights into molecular mechanisms of diffuse coronary ectasiae: a possible role for VEGF. Int I Cardiol 2006; 106: 307-12.

14. Lassalle P, Molet S, Janin A, et al. ESM-1 is a novel human endothelial cell-specific molecule expressed in lung and regulated by cytokines. J Biol Chem 1996; 271: 20458-64.

15. Tunick PA, Slater J, Kronzon I, et al. Discrete atherosclerotic coronary artery aneurysms: a study of 20 patients. J Am Coll Cardiol 1990; 15: 279-82.

16. Markis JE, Joffe CD, Cohn PF, et al. Clinical significance of coronary arterial ectasia. Am J Cardiol 1976; 37: 217-22.

17. Balta S, Ozturk C. The relation between CD40 ligand and coronary artery disease. Cardiology 2015; 131: 107-8.

18. Shin JW, Huggenberger R, Detmar M. Transcriptional profiling of VEGF-A and VEGF-C target genes in lymphatic endothelium reveals endothelial-specific molecule-1 as a novel mediator of lymphangiogenesis. Blood 2008; 112: 2318-26.

19. Balta S, Mikhailidis DP, Demirkol S, et al. Endocan: a novel inflammatory indicator in cardiovascular disease? Atherosclerosis 2015; 243: 339-43.

20. Markis JE, Joffe CD, Cohn PF, et al. Clinical significance of coronary arterial ectasia. Am J Cardiol 1976; 37: 217-22.

21. Sanad O, Al-Keshk E, Ramzy A, et al. Characteristics of coronary artery ectasia and its association with carotid intima-media thickness and high sensitivity C-reactive protein. Atherosclerosis Suppl 2017; 25: e2-3.

22. Li JJ, Nie SP, Qian XW, et al. Chronic inflammatory status in patients with coronary artery ectasia. Cytokine 2009; 46: 61-4.

23. Turan T, Akyuz AR, Aykan AC, et al. Plasma endocan levels in patients with isolated coronary artery ectasia. Angiology 2016; 67: 932-6.

24. Soto ME, Reyes-Villatoro MA, Márquez R, et al. Evaluation and analysis of plasma soluble adhesion molecules in patients with coronary ectasia and atherosclerotic coronary artery disease. Arch Med Res 2014; 45: 478-83.

25. Turhan H, Erbay AR, Yasar AS, et al. Plasma soluble adhesion molecules; intercellular adhesion molecule-1, vascular cell adhesion molecule- 1 and E-selectin levels in patients with isolated coronary artery ectasia. Coron Artery Dis 2005; 16: 45-50.

26. Cai W, Schaper W. Mechanisms of arteriogenesis. Acta Biochim Biophys Sin 2008; 40: 681-92.
27. Paulus P, Jennewein C, Zacharowski K. Biomarkers of endothelial dysfunction: can they help us deciphering systemic inflammation and sepsis? Biomarkers 2011; 16 Suppl 1: S11-21. 